\title{
Perspectivas antropológicas sobre las formas de (ganarse la) vida
}

\author{
Anthropological perspectives on forms of (making a) living \\ Perspectivas antropológicas sobre formas de (ganhar a) vida
}

\author{
1 Universidad de Buenos Aires. Ciudad de A. de Buenos Aires, Argentina \\ Correo electrónico: mifernandezalvarez@gmail.com \\ ORCiD: 0000-0003-4201-2694 \\ 2 Universidad de Buenos Aires. Ciudad de A. de Buenos Aires, Argentina \\ Correo electrónico: mdperelman@gmail.com \\ ORCiD: 0000-0002-4914-3198
}

\section{Introducción}

La reflexión sobre las maneras en que las personas producen formas de garantizar aquello que consideran una vida digna ha cobrado relevancia en los estudios antropológicos, especialmente a la luz de las transformaciones del capitalismo contemporáneo de las últimas décadas. En el norte global, especialmente en Europa, esta reflexión tomó especial dinamismo con la crisis financiera de 2008, que puso en evidencia procesos de más largo aliento vinculados al desmantelamiento del estado de bienestar y las formas de precarización de la vida de amplios sectores de la población. La literatura antropológica buscó así elaborar categorías analíticas que permitieran abordar las formas en que las personas hacen frente a estos procesos.

Una de las reflexiones más sugerentes en esta dirección fue formulada por Susana Narotzky y Niko Besnier en un número especial de Current Anthropology publicado en el año 2014, cuya traducción al español abre este número temático de la Revista Cuadernos de Antropología Social. Allí, elaboran una propuesta programática que, sorteando los límites de los modelos abstractos, nos posibilita "repensar la economía" al colocar la mirada en las continuidades y transformaciones de los sistemas colectivos que permiten sostener la vida. Esto incluye, desde su perspectiva, las posibilidades y necesidades objetivas y subjetivas de las personas para proyectar su vida futura. Recuperan para ello contribuciones de la economía política, la economía moral y la economía feminista, y proponen en su articulación una perspectiva que apuesta a formular una teoría antropológica de la reproducción social en el capitalismo actual.

Con este propósito se valen de la potencialidad que tiene la etnografía para desarrollar un abordaje "ordinario" acerca de la naturaleza de la vida económica, que nos invita a poner en el centro de nuestro análisis la diversidad de prácticas y procesos involucrados

Nota de los autores: Quisiéramos expresar nuestro profundo agradecimiento a Sandra Wolanski por el cuidadoso trabajo de seguimiento, edición, comunicación y lectura de los textos y de la presente introducción, realizado a lo largo de todo el arduo trabajo editorial que implica la publicación de un dossier. Sin su trabajo, este número no hubiese sido posible. También queremos agradecer el trabajo editorial por parte de la revista -en especial a Julieta Infantino y a Cecilia Benedetti-, que en el contexto de aislamiento social han tenido templanza y paciencia para acompañar el trabajo que todes les autores y evaluadores han realizado. Nos sentimos profundamente agradecides. 
en las formas de "ganarse la vida" y de construir "vidas que merecen ser vividas". Nos advierten, al mismo tiempo, sobre la importancia de atender tanto al carácter histórico y culturalmente situado que cobran esas formas de ganarse la vida en contextos específicos, como a la relación entre modelos abstractos y prácticas cotidianas, lo que implica afirmar la materialidad de dichos modelos y los efectos sobre conjuntos amplios de la población. Sobre la base de un procedimiento tan propio como productivo del modo de producción de conocimiento antropológico -la elaboración de categorías analíticas a partir de nociones de la práctica-, el artículo publicado originalmente en Current Anthropology introduce así una nueva lente analítica desde la que colocar la mirada sobre la forma en que las poblaciones sostienen la vida, no solo material, sino también social, afectiva, política, etcétera.

Una preocupación semejante motiva la invitación a la que nos convoca Benoît de L'Estoileen su texto "El dinero es bueno, pero un amigo es mejor". Incertidumbre, orientación al futuro y "la Economía", que acompaña la convocatoria del presente número temático. De manera sin duda provocadora, el antropólogo francés propone allí abandonar la idea de "economía" al señalar la potencia del enfoque etnográfico para desarrollar, no solo una perspectiva plural de las prácticas económicas, sino también, y sobre todo, una mirada atenta a las formas situadas de construir el mundo y de vivir en él. Otras formas de vida, más que otra economía. El término oikonomia o "gobierno de la casa", afirma el autor, permite reconceptualizar las prácticas generalmente vistas como parte de la "esfera doméstica", al subrayar a los aspectos políticos, morales y afectivos involucrados en las formas cotidianas para sostener la la vida. Así, su análisis introduce y prioriza la política en el estudio de las formas de (re)producir la vida generalmente asociadas a "la economía".

En síntesis, ambas nociones - "ganarse la vida" y "formas de vida"- constituyen una puerta de entrada para repensar "la economía" y discutir su alcance, que reivindica la productividad de una comprensión holística de la vida social. Este número temático de la revista Cuadernos de Antropología Social se vale de la ruta trazada en ambos textos en la apuesta por integrar aquello que, como producto de la reproducción de campos (sub) dsiciplinares, suele quedar reducido a dimensiones de análisis "económicas", "políticas" , "culturales", "sociales", "morales". Este es, pues, el espíritu que organiza la propuesta y reúne los artículos incluidos en el presente dossier.

\section{Formas de (ganarse la) vida: afirmar la totalidad}

Hablar de comprensión holista en antropología puede resultar, paradójicamente, algo tan evidente como materia de crítica en la medida en que ella ha estado asociada a una idea de organicidad especialmente presente en los estudios clásicos. Vale preguntarnos si no es posible encontrar en esta paradoja una de las claves para comprender por qué, tratándose de uno de los principales atributos de nuestra disciplina, resulte a la vez una característica las más de las veces ausente en nuestras etnografías. Allí radica, pues, uno de los objetivos de este número temático: reflexionar sobre el potencial de nociones como "ganarse la vida" o "formas de vida" en tanto categorías analíticas que permitan (re)incorporar un análisis holístico a los estudios sobre temas tan diversos como la vida de artistas independientes, vendedores ambulantes, costureras o personas que obtienen sus ingresos a partir del robo, el acceso de sectores populares al crédito, las demandas por la autonomía social y política, entre otras. Atender a las formas de (ganarse la) vida permite, así, no partir de un "campo" preestablecido de problemas, sino mostrar la complejidad que constituyen los procesos sociales de forma holística, más allá de recortes apriorísticos. 
En los textos que ofician de apertura del dossier y organizan la propuesta, la apuesta por una comprensión holística se aleja de toda herencia organicista para abrazar, en cambio, desde diferentes perspectivas analíticas, la idea de totalidad. De ahí que cobren relevancia nociones como reproducción social, sobre la cual la contribución de Gavin Smith introduce una (re)lectura sumamente iluminadora. Formular una teoría etnográfica de la reproducción social en el capitalismo actual es, en efecto, el objetivo último de la tarea que emprenden Susana Narotsky y Niko Besnier. Tal como lo señalan en su artículo, esta categoría teórica cobra suma relevancia en la medida en que permite desarrollar una metodología de escala que busca cuestionar la separación entre modelos "abstractos" y "manifestaciones concretas", a la vez que evidencia el componente ideológico -y performativo- de esa separación como parte las formas de acumulación capitalista. ${ }^{1}$ Pero se trata de desarrollar una perspectiva de la reproducción social capaz de hacer confluir este análisis de escala con un estudio cuyo foco esté puesto en las prácticas concretas de la vida diaria. De ahí que les autores propongan definir la reproducción social como "una continuidad que une a las generaciones en torno a microproyectos para ganarse la vida y mejorar sus oportunidades futuras, y macroproyectos de configuraciones sociales de poder y distribución de recursos" (ver en contexto).

Reproducción social es, pues, un término sustantivo en pos de un enfoque holístico que toma distancia de una mirada organicista y pone el foco en la idea de totalidad. Mientras que las reflexiones de Susana Narotzky y Niko Besnier y de Gavin Smith se nutren de este concepto, cuyas raíces son de economía política y los aportes del feminismo, Benoît de L'Estoileretoma la noción dual de Reinhart Koselleck de "espacio de experiencia" (Erfarhungsraum) y "horizonte de expectativa" (Erwartungshorizont), en diálogo con la noción de campo de oportunidades elaborada por Max Weber. Con este último concepto, a partir de una etnografía de larga duración en la zona de Pernambuco, al nordeste de Brasil, analiza el "conjunto de posibilidades y restricciones en un momento dado las 'condiciones para la vida', tanto materiales como simbólicas" (ver en contexto).

Larga duración es un término que remite a una mirada que merece ser destacada y señala un aspecto sumamente relevante que nos interesa enfatizar en la apuesta por un enfoque holístico que se aleje de toda herencia organicista. La incorporación en el análisis etnográfico de los procesos históricos y la forma en que estos configuran y modelan de manera situada formas de vida o de ganarse la vida deviene insumo clave de la tarea a la que nos convocan. La historia es texto que da contenido no solo a las condiciones materiales de vida sino también a los contornos que cobra la forma en que se define una vida que vale la pena ser vivida y las expectativas o proyectos a futuro. El análisis que emprende Gavin Smith en torno a su estudio con trabajadores rurales en Perú y España -dos contextos y momentos distantes: la zona andina durante la década del setenta en el primer caso, la comunidad valenciana en la década del ochenta en el segundo- pone en evidencia la fertilidad de una perspectiva etnográfica históricamente informada. Los artículos de Julieta Gaztañaga, Hadrien Saiag y Lenin Pires brindan igualmente pistas sobre la forma en que nuestras etnografías se enriquecen al hacer parte de nuestro análisis una reconstrucción histórica situada. Estos trabajos, además, nos recuerdan la importancia de la mirada comparativa de los procesos sociales.

Emprender una comprensión holista supone casi de manera intrínseca una idea de integración, imbricación o entrelazamiento, para usar los diferentes términos a los que apelan el conjunto de los textos reunidos en el dossier a la hora de evidenciar los límites de abordajes que separan la vida social en dimensiones "económicas" "políticos" "afectivas" "morales", etc. Como es evidente, lejos está de ser un aspecto que podamos considerar novedoso constituyendo una de las principales contribuciones de la antropología respecto de otras miradas disciplinares. Los textos aquí reunidos,
1. En este contexto, Neiburg y Guyer (2019) plantean la necesidad de pensar la "economía real", tanto como una categoría técnica, así como una forma de expresar procesos a partir de sus usos vernáculos. 
2. Cuando abrimos la convocatoria para el dossier recibimos un número inesperado de artículos de calidad que por cuestiones de espacio no pudimos incluir. Estos textos abarcan diferentes casos, temas y regiones. Ello, creemos, da cuenta de la pertinencia teórica de la perspectiva a la que este número temático busca contribuir. Con este objetivo, buscamos incluir contribuciones sobre una diversidad de tematicas y regiones que den cuenta de estas diferentes miradas de las formas (de ganarse la) vida. sin embargo, logran ir más allá de un trabajo analítico que consiste en evidenciar el carácter imbricado de la vida social: convierten este punto de partida en perspectiva para. Más que mostrar que la vida social es total, hacen de ese atributo un camino para discutir nociones como trabajo, soberanía, política, crédito, dinero, estado y ponen en el centro el "esfuerzo por hacer vida", "producir personas" para usar los términos que utilizan Susana Narotsky y Niko Besiner; “construir el mundo y vivir en él”, en palabras de Benoît de l'Etoile. ${ }^{2}$

Este dossier se propone explorar la potencialidad de estas categorías analíticas -formas de vida, ganarse la vida, a la que sin duda podemos agregar modos de vida a la que apela Gavin Smith-en tanto perspectiva holística que permita colocar la mirada en la forma en que las personas producen y dan sentido a la vida como totalidad. Esto implica incluir en esa producción tanto aquello que hacen para asegurar su reproducción en términos materiales como las formas en que definen una buena vida, es decir, una vida que merece ser vivida o una vida digna (Fernandez Alvarez, 2017; Fernandez Alvarez, 2020a; Fernández Álvarez, Wolanski, Señorans, Pacifico et. al, 2019). Es en esta clave que nos interesa poner en diálogo los artículos reunidos en este dossier a partir de una puesta en común que nos permita explorar las posibilidades de ampliar esta reflexión en dos direcciones complementarias. Por un lado, interrogar su alcance para trascender un recorte definido en términos de "clase" o "sector social" vinculado a determinadas poblaciones definidas como "pobres", "precarias", "marginadas" o "excluidas". Esto no quiere decir, sin embargo, dejar de lado un análisis que contemple las formas específicas que en contextos situados desarrollan las personas para sostener la vida, retomando el señalamiento que realiza Gavin Smith en su contribución, lo que implica reconocer los procesos de desigualdad social y los procesos de explotación. Por otro lado, buscamos indagar las posibilidades de pensar esta perspectiva más allá de una discusión ligada a la noción de "crisis" para explorar su potencialidad en una diversidad más amplia de contextos y situaciones atendiendo a las especificidades regionales y locales.

\section{Una mirada global desde el sur}

Si en los últimos años los estudios sobre las formas de (ganarse la) vida, han estado signados por rupturas e incertidumbres, la historia latinoamericana nos pone en la necesidad de revisar la temporalidad de estos procesos y recuperar la larga tradición que tienen las investigaciones en la región.

Los procesos latinoamericanos nos exigen poner entre paréntesis, al menos, la necesidad de pensar en términos analíticos la noción de crisis. En primer lugar, porque resulta necesario indagar en qué implican o qué son las rupturas en las expectativas, los reordenamientos de valores y marcos de obligaciones morales en una región que constantemente ha vivido en "crisis". También porque cada país, y cada región, tiene temporalidades propias (recordemos aquí la hiperinflación de 1989 o la crisis de 2001; el impacto de las crisis financieras y las devaluaciones de 1994 o 1999 en México y Brasil, o de la crisis global de 2008, de una forma muy diferente a la que dio fuerza a los debates antropológicos de la crisis en el "norte global”). En este marco, una mirada desde América Latina puede poner en cuestión los usos de dicha noción para describir procesos de pauperización y precarización. Por ejemplo, se suele decir que los pobres son resilientes (que "siempre viven en crisis"), postura que solo corrobora la visión clase-céntrica de ciertas utilizaciones de ese concepto para pensar las "consecuencias" de los procesos sociales.

No debemos olvidar, sin embargo, que "crisis" es una categoría nativa que los actores movilizan en contextos y situaciones determinadas. Así una mirada desde Latinoamérica nos obliga a pensar las dimensiones particulares que producen esas vidas largamente 
desiguales y precarias. Y requieren que estemos atentos a los usos que los grupos sociales hacen de las categorías para inscribir procesos sociales específicos y locales.

Una perspectiva holista de larga duración nos permite pensar la persistencia de las desigualdades en América Latina, las múltiples agencias y maneras en que las personas dotan de sentido a su vida, y el modo en que esas vidas forman parte de un entramado social y productivo.

En esta región, las formas de vivir que pueden considerarse "inciertas" cuentan con una larga historia. $Y$ es justamente esa larga experiencia la que permite comprender, no solo la construcción social de las formas de incertidumbre -que no siempre están ligadas a las "crisis económicas"-, sino también las propias miradas teóricas y metodológicas de América Latina. Recordemos, por ejemplo, el surgimiento de la teorías de la dependencia (Cardoso y Faletto, 1969; Gunder Frank, 1970; Marini, 1973), y nociones como marginalidad (Germani, 1973), polo marginal (Quijano, 2014), masa marginal (Nun, 2001), que buscaron explicar el acceso a la vida de miles de personas que estaban fuera del mercado de trabajo. ${ }^{3}$

En síntesis, nos interesa señalar que han existido aportes sustantivos de la tradición latinoamericana que han buscado pensar los modos de sobrevivir y las formas de integración social y de ciudadanía en el continente más desigual del mundo. Así, desde mediados de la década del setenta y especialmente a partir de los procesos de reforma neoliberal, una serie de investigaciones han mostrado cómo -especialmente en contextos de pobreza, desempleo y precarización social- son las redes territoriales, familiares, de amistad o reciprocidad, así como las diferentes formas de intervención estatal y de ONG o los procesos de movilización, las dinámicas de organización social y los proyectos colectivos, los que configuran un complejo entramado para sostener la vida. En su conjunto, estos trabajos han permitido ponderar la capacidad de agencia de los sectores populares.

En esta tradición la obra célebre de Larissa Adler de Lomnitz, Cómo sobreviven los marginados, (1975), y su crítica a la visión de Oscar Lewis (1969) sobre la cultura de la pobreza y las nociones de marginalidad a lo Germani constituye una referencia incontournable. La autora parte de la noción de marginalidad y señala allí que en las barriadas que estudia eran básicamente las redes de reciprocidad (parentesco, vecindad, compadrazgo, amistad) las instituciones centrales. Más cercano en el tiempo, las nociones de estrategias (de vida, supervivencia, adaptativas, de reproducción social) también buscaron generar conocimiento en esta línea. La noción de acumulación de desventajas, desarrollada por González de la Rocha (2004) para el caso de México en el contexto de la implementación de políticas a partir del "Consenso de Washington", constituye otro aporte de relevancia de la tradición como nuevas formas de entender la vida desde América Latina (ver Saraví, 2007 para otros países latinoamericanos).

En resumen, todos estos estudios han puesto de relieve la realidad de miles de personas en América Latina. Lo cierto es que estos procesos de larga duración han dado cuerpo a una serie de formas de entender la vidas precarias desde América Latina -que ha sido una suerte de laboratorio de políticas que generaron pobreza-. Además las tradiciones de importantes centros de producción antropológica en la región han dado cuenta de las múltiples formas en que los procesos de precariedad no pueden entenderse solo como dinámicas abstractas, sino como formas locales de entender la explotación, de vivir, de resistir, de demandar.

Si bien gran parte de estas pesquisas han sido territoriales -o sea, focalizadas en el locus de la pobreza- tal como lo que venimos desarrollando en estudios previos (Cosacov y Perelman, 2015; Perelman, 2015; Fernández Álvarez, 2017, 2020a; Perelman y Boy,
3. Para un análisis de las teorías del desarrollo y la pobreza en América Latina ver Álvarez Leguizamón (2008). 
4. Entre otros textos, cf. Kashmir y Carbonella (2008), Carrier y Kalb (2015), Fernández Álvarez (2018, 2020b), Kashmir (2018), Millar (2017), Sanchez (2018), Lazar y Sanchez (2019), Wolanski (2020).
2017), nuestra propuesta busca salir de espacios estancos para mostrar las múltiples espacialidades y procesos ligados a la producción y reproducción de modos de vida precarios, pero más allá de la precariedad. Las estrategias de supervivencia, las desventajas acumuladas o el mero análisis de los sectores populares en sus lugares de morada iluminan ciertos aspectos de la vida social. Pero una mirada que dé cuenta de las interacciones, de las formas de trabajo en las fábricas, en la calle, en los negocios; el estudio de las acciones políticas en diferentes escalas nos permiten iluminar las formas de vivir más allá de la supervivencia o de la obtención de recursos materiales.

Poner en diálogo las contribuciones desarrolladas desde América Latina con la literatura producida en otros contextos académicos permite recuperar y poner de relieve formas de entender el mundo desde diferentes tradiciones teóricas. Si, como dijimos, los procesos de crisis iniciados en 2008 han dado un nuevo aliento a los debates en torno a las formas en que las personas producen formas de garantizar aquello que consideran una vida digna, este dossier busca contribuir a pensar estas formas, no solo a partir de la pérdida de un estilo de vida que se pone en jaque y genera incertidumbre, sino también a partir de largos procesos de pauperización, precariedad y desigualdad.

\section{Trabajo(s), valor(es) y futuro(s)}

Los artículos aquí reunidos ponen de relieve el modo en que colocar en el centro de nuestro análisis las formas en que las personas producen una vida que consideran digna permite ampliar nuestra compresión sobre el trabajo, las formas de producir valor y el futuro.

Detengámonos en primer lugar en revisar la forma en que los artículos contribuyen a ampliar nuestro abordaje sobre el trabajo, un término que recorre la totalidad de los textos y sin duda constituye una de las categorías que a priori asociamos de manera más directa al hablar de formas (o modos) de (ganarse la) vida. Sin ánimo de proponer aquí una suerte de actualización sobre las reflexiones antropológicas actuales en torno a esta temática, vale la pena señalar que en los últimos años, y a la luz de las transformaciones en el capitalismo tanto en el norte como en el sur global, los debates en la disciplina sobre este campo se han visto renovados, y nos invitan a repensar, por ejemplo, las nociones de clase o de precariedad. ${ }^{4}$ En nuestra región, la creación de una revista dedicada a la temática, como es el caso de la Revista Latinoamericana de Antropología del Trabajo, así como la multiplicación de espacios en este campo en eventos académicos, refuerzan esta idea.

Como lo evidencian los artículos aquí reunidos y como lo hemos desarrollado en trabajos previos (Fernández Álvarez, 2016, 2017, 2020a; Perelman, 2016, 2017, 2020), un aspecto sustantivo de esta renovación consiste en proponer un desplazamiento de una antropología del trabajo que privilegia un abordaje sobre la relación salarial -e incluso el mundo obrero-, para colocar la mirada en la heterogeneidad y multiplicidad de las formas de trabajo que recuperan la cotidianeidad social en el que esas prácticas adquieren sentido. Resulta evidente, nuevamente, que la antropología ha dado históricamente cuenta de esta cuestión; basta con mencionar aquí la obra célebre de Eric Wolf, Europa y la gente sin historia, en la que insiste sobre la necesidad de estas formas múltiples de trabajo para la producción de plusvalía (Kashmir y Carbonella, 2008). La renovación de las reflexiones antropológicas sobre este campo han ido así de la mano de una búsqueda por repensar el concepto de trabajo (Narotzky, 2018) en un esfuerzo por ampliar nuestra mirada que incluya no solo las modalidades no asalariadas, sino también aquellas no mercantilizadas. En relación con esta cuestión, ha sido sin duda sustantivo el aporte de las perspectivas feministas que desde hace décadas vienen 
insistiendo en una reflexión crítica de la diada trabajo productivo-reproductivo, que ha cobrado fuerza en las reflexiones antropológicas y en las ciencias sociales en general.

Así, un primer aspecto de esta renovación podría sintetizarse en un desplazamiento de la noción de trabajo a la de modos de vida, para colocarlo en los términos que lo propone Gavin Smith. Este desplazamiento implica incorporar en nuestro análisis tanto las relaciones mercantiles que tienen lugar en el mercado de trabajo -formal o informal-, pero también toda una multiplicidad de prácticas y relaciones no medidas por el mercado pero que son sustantivas para (re)producir la vida, como lo evidencian los textos de Hadrien Saiag, Julieta Quirós, Mariana del Mármol, Dolores Señorans, Lenin Pires y Pablo Figueiro. Aquí, cobran centralidad vínculos de amistad o militancia que garantizan el acceso a un trabajo remunerado, como en el caso de los artistas que se dedican al trabajo independiente en La Plata, al que se aboca Mariana del Mármol; o las relaciones de parentesco, vecindad y procedencia migratoria que permiten, no solo sostener la vida, sino proyectar una vida mejor, como lo analiza Dolores Señorans en su estudio sobre formas de organización gremial de trabajadores costureros en Buenos Aires. Las relaciones de amistad y parentesco también cobran relevancia en el análisis que propone Pablo Figueiro sobre los sentidos del trabajo, la vida y el dinero para personas que se encuentra privadas de su libertad por prácticas delictivas. Su análisis pone de relieve la centralidad de estos vínculos en las concepciones y experiencias de aquello que se define como vida digna, y la forma en que en ese contexto se significa el delito como modo de ganarse la vida. Al analizar la venta ambulante en Buenos Aires y en Río de Janeiro, Lenin Pires muestra cómo las actividades que permiten obtener dinero no pueden entenderse solo desde el "trabajo", sino a partir de los procesos de regulación social más allá del eje entre el eje legal-ilegal. El trabajo aquí puede ser pensado como una forma de relación social que se entiende desde una mirada de los intereses de los actores en la sociedad y de las sensibilidades jurídicas que lo posibilitan. Como lo muestran los textos de Saiag, Señorans o Quirós, este desplazamiento supone un descentramiento de los lugares definidos como "espacios de trabajo" a los hogares, los barrios, los espacios comunitarios.

Este desplazamiento trae consigo un segundo aspecto que nos interesa destacar en particular. Al mostrar la centralidad que cobran las relaciones no mercantiles, nuestro análisis pone en evidencia que la vida se sostiene a partir de vínculos de interdependencia que cuestionan un ideal de autonomía vinculado al trabajo formal (asalariado, masculino, blanco, etc.), un aspecto sobre el que las perspectivas feministas también han sido pioneras. En efecto, insistir sobre la importancia de visibilizar las tareas reproductivas y de cuidados -realizadas fundamentalmente por mujeres- ha permitido no solo mostrar el carácter productivo de estas actividades y destacar su contribución en el proceso de acumulación (Benería, 1987; Frederici, 2010), sino también ampliar la noción de trabajo señalando la totalidad de procesos y relaciones de interdependencia que posibilitan la (re)producción de la vida en un sentido no solo material sino afectivo y emocional (Carrasco, 2013; Pérez Orozco, 2014; Comas d’Argemir, 2015). Los artículos de Mariana del Mármol y Dolores Señorans a los que hicimos referencia previamente dan cuenta a la vez de los vínculos de interdependencia y el carácter inescindible entre materialidad, emocionalidad y afecto en las formas de (ganarse la) vida, así como también contribuyen a discutir otras dicotomías como individual/colectivo. En particular, el análisis sobre los procesos de organización gremial de costureros en el AMBA que elabora Dolores Señorans señala los límites de una perspectiva que opone aspiraciones de progreso individual a la apuesta por construir formas de bienestar colectivo, para destacar en cambio el carácter plural de las economías populares. Trabajar en común es la fórmula que propone Julieta Quirós para discutir esta ecuación que hilvana labores individuales en tareas colectivas que convierten a sus participantes en coproductores y-como tales- en copropietarios de bienes comunes. El análisis que propone la autora destaca cómo, a partir de ese trabajo, lo que se produce no es tanto 
5. Sobre estos temas han surgido varios números temáticos en revistas y libros. Ver, por ejemplo, el sitio $\mathrm{Hau}$, desde donde que ha dado un intenso impulso a estas cuestiones con la publicación de su revista y de sus libros.

6. Es posible decir que un camino inverso ha sido realizado por los estudios sobre el dinero, las finanzas y (de) la economía, que han contribuido a mostrar el carácter

social de la producción de valor allí donde este parece ser "natural". "comunidad" sino "partes comunes" - a la vez múltiples y diferenciadas- del "cuerpo social y sus procesos de vida".

Una segunda cuestión que nos interesa puntualizar es el modo en que los textos aquí reunidos contribuyen a pensar la noción de valor(es). Así como la noción de trabajo, la de valor también ha tenido en los últimos años un renovado interés en nuestra disciplina, de la que dan cuenta en su artículo de Susana Narotzky y Niko Besnier. Recientemente, diferentes perspectivas y corrientes han resituado la pregunta sobre el "valor" como eje de sus indagaciones y reflexión teórica (números de Hua Journal of Ethnographic Theory, 3 (1 y 2); Gregory, 2015; Narotzky, 2016; Robbins y Stein, 2016). Esta renovación se vio alimentada principalmente por debates en torno a las éticas (Lambek, Das, Fassin y Keane, 2015), el giro ontológico (Kohn, 2015; Heywood, 2017) y los debates en torno al antropoceno (Chua y Fair, 2019; Mathews, 2020), ${ }^{5}$ a los estudios sociales del dinero y las finanzas (Parry y Bloch, 1989; Maurer, 2006; Neiburg, 2010; Hart y Ortiz, 2014; Guyer, 2016), ${ }^{6}$ entre otros. Estos debates buscan pensar la producción de valor en términos económicos, pero sobre todo, las múltiples formas en que se produce y otorga valor en un sentido amplio. Para eso es importante indagar aquello que emerge como valioso en distintos casos etnográficos ("valor" como lo muestran Narotzky y Besnier).

El capitalismo como forma de producir valor nos invita a pensar en estas formas generales (y supuestamente homogéneas) de valores económicos y también de formas de vida. La mirada etnográfica, como un antídoto a los lugares comunes, nos permite mostrar la producción de valor y de esferas de valor, así como las relaciones y conversiones que se producen entre diferentes esferas de valor. El texto de L'Estoileavanza en esta línea al plantear no sólo la dificultad de pensar en términos de "economía", sino también con la peligrosidad de confundir los "lenguajes". Así, nos dice que es probable que nuestros marcos (como investigadores) nos engañen al plantear prácticas que utilizan el lenguaje económico como "trabajo", "salario", "venta", "dinero", "ir al mercado" y "mantener la casa", refieran principalmente a lo "económico"; hablan sobre cómo vivir y vivir bien. Amigos y dinero en este sentido no refieren a una misma esfera de valor sino también a pensar el modo en que en contextos de incertidumbre las personas producen valoraciones diferentes que no pueden reducirse a lo económico.

Pensar en términos de valor no solo implica pensar en los actores "racionales" que se encuentran en un mercado para intercambiar productos. La producción de valor en relación con los modos de ganarse la vida implica pensar el valor económico, pero también:

El interés en lo que realmente le importa a las personas de todo el mundo y en cómo las culturas difieren no solo como sistemas de poder, producción o significado, sino como esquemas que ayudan a definir lo que en última instancia es bueno y deseable en la vida. (Robbins y Stein, 2016, p. 8)

En este marco, una de las líneas centrales que creemos importante recuperar es la relevancia que en la actualidad tiene una perspectiva que no solo se centre en la noción de valor desde la "economía". Esto no quiere decir que debemos desechar este camino. Antes bien, creemos que -acompañando un creciente campo de estudio sobre el sistema financiero y el dinero- se hace necesario pensar el modo en que incluso en el corazón más "puro" de la "economía" son los procesos históricos, las relaciones sociales y las motivaciones, moralidades, acciones plurales, como plantea Señorans, de las personas en función de sus prácticas y las relaciones de poder las que construyen el valor. Es esta misma idea la que busca poner en relieve Gavin Smith en su crítica a las formas de ver las vidas y la "economía". La noción de livelihoods permite mostrar las acciones a partir de la producción de valoraciones sociales de la vida más allá de la economía. 
En Argentina, el creciente estudio de los procesos de financiarización de los sectores populares y el acceso al consumo comienzan a dar cuenta de la experimentación de los usos del dinero, la deuda y los procesos de valorización social que se producen a partir de la multiplicidad de piezas monetarias, así como del consumo. El texto de Saiag muestra el modo en que un punto clave en este proceso es el boom del crédito al consumo destinado a personas con trabajo precario. Su artículo señala cómo el acceso al crédito permite una valorización del consumo como una forma de inclusión, al tiempo que genera una mayor precarización. El crédito genera acceso a nuevas formas de consumo y somente, al mismo tiempo, a los deudores a formas de explotación también novedosas.

El abordaje etnográfico de las diferentes formas de producción de valor no implica desconocer el poder que tienen los saberes expertos -aquello que podríamos plantear como "los modelos abstractos" en los términos que desarrollan Narotzky y Besnier en el texto que abre el dossier- en la producción del valor y en el modo en que las personas "ordinarias" construyen formas dignas de vivir. Ello, entendemos, no tiene que ser un punto ciego ni dado de las investigaciones, sino objeto de indagación. Este camino permite desentrañar la dicotomía imaginaria -aunque bien real- entre acciones irracionales y racionales: mientras la mirada experta sería racional, la de las personas ordinarias, no. Esta diferenciación no solo impide ver la coconstrucción de las formas de producir valor en el mundo, sino también comprender los diferentes órdenes y esferas de valor que constituyen la vida social.

Retomando las contribuciones que David Graeber (2018) ha realizado sobre la idea de valor, el análisis de Gaztañaga en su etnografía con activistas que trabajan para instalar el derecho a decidir sobre el estatus político del pueblo vasco muestra la producción social del soberanismo como valor; siendo el "valor" entendido como la atribución de importancia a las acciones por parte de sus protagonistas, al ser incorporadas en una totalidad social más amplia. Desde este enfoque, la valorización es un proceso de realización cotidiano que implica siempre la producción de alguna forma de totalidad social imaginaria, como en este caso el soberanismo democrático en tanto proyecto de construcción estatal alternativo. Algo similar podemos decir del texto de Julieta Quirós. En su etnografía, la autora atiende al flujo de energía social que las comisiones vecinales invierten diariamente en producir y reproducir sus condiciones materiales de existencia, basado en formas de entender y mantener la vida. Ello se basa en una serie de valoraciones sobre cómo se vive que no pueden pensarse como "políticas" o "económicas".

Pensar el valor implica también indagar en las valoraciones, como lo hace Lenin Pires en torno a las sensibilidades jurídicas y los usos del "derecho" en situación. De esta forma, ganarse la vida en la calle implica negociaciones y disputas entre las mismas agencias en el ejercicio de control sobre el público en general; lo cual puede abrir espacios entre las estructuras normativas y de poder. A partir del trabajo de Pires, es posible pensar la producción de valor en relación con el "derecho", con la "economía" y con los propios sentidos que las personas otorgan a sus vidas. Es por ello que ciertos procesos, como la violencia y la falta de respeto, aparecen como formas de desvalorización. Como plantea uno de sus interlocutores, dejar la venta "no es solo una cuestión de plata".

La cuestión del valor abordado desde una perspectiva antropológica permite pensar en los modos en que se fijan precios -ya sea en el "mercado", o en términos de precio justo, más ligados a la economía moral-, pero también a acciones cotidianas, modos de vivir, formas de entender el trabajo o la política. Las elecciones morales sobre cómo ganarse la vida implican modos de valorar el ingreso de dinero, pero también ponderar formas de sociabilidad, militancia o relaciones afectivas, como demuestran los trabajos de Del Mármol, Figueiro y Pires. En esta misma línea, es posible plantear que las personas resignifican ciertas formas sociales de valor para poder construirlas como 
7. Para una revisión crítica sobre la forma en que la antropología abordó el futuro como objeto de indagación cf. Pels (2015), Pink y Salazar (2017), Bryant y Knight (2019), Visacovsky (2019). un modo de vida digna, como en el caso analizado por Luis Acatzin Arenas Fernández. Su trabajo muestra que la danza "cultural" permite la circulación de efectivo, que se torna una forma de "ganarse la vida". Pero al mismo tiempo, a partir de la lógica neoliberal y multicultural, se abren para los actores espacios en el mercado, formas ambiguas de dar valor a la vida, y la construcción de expectativas optimistas a pesar de la incertidumbre en que viven.

Finalmente, un último tópico recorre los artículos reunidos en este número temático que ha cobrado relevancia en los últimos años vinculado a las reflexiones sobre la noción de futuro. ${ }^{7}$ Como lo señala Sergio Visacovsky (2019), dentro de este campo de conformación reciente se destacan las reflexiones en torno a las nociones de esperanza e incertidumbre, dos términos centrales en los artículos de nuestros autores invitados que inauguran el dossier. Mientras que el texto de Susana Narotsky y Niko Besnier se detiene particularmente en el primero, la noción de incertidumbre recorre el texto de Benoît de L'Estoilelo hace en una reflexión que se vale de la polisemia del término esperar en portugués - $\mathrm{y}$ en español- para explorar la forma en que sus interlocutores hablan sobre sus visiones del futuro. Así, esperanza e incertidumbre operan en ambos casos como términos complementarios que ponderan una dimensión proyectual -a la vez en sentido de proyecto y proyección- en el análisis sobre las formas de (ganarse la) vida. Lejos de ser una potestad de los modelos económicos, el largo plazo, la inversión a futuro, las formas de imaginar una vida que merece ser vivida hacen parte de la forma en que en el día a día las personas sostienen y producen formas de (ganarse la) vida. Así, esta lectura pone en cuestión una idea de corto plazo e inmediatez que suele estar asociada, por ejemplo, a las prácticas orientadas a la reproducción de la vida principalmente cuando se trata de sectores populares. En efecto, al desplazar la mirada de las prácticas "económicas" a las formas en que las personas construyen vidas que merecen ser vividas -como proponen Susana Narotzky y Niko Besnier- o las condiciones y medios necesarios para "vivir y vivir bien" -siguiendo a Benoît de l'Estoile-, esta perspectiva contribuye a mostrar la capacidad de imaginar, proyectar y planificar de poblaciones que suelen ser caracterizadas como movidas por la urgencia del día a día.

En síntesis, ganarse la vida, formas de vida, modos de vida, son categorías que se inscriben en una perspectiva que va más allá del orden de la supervivencia y lo inmediato (aunque sin duda lo incluyen), para hacer lugar a las orientaciones y proyecciones a futuro. Así lo evidencia el análisis que propone Hadrien Saiag sobre las prácticas financieras de los sectores populares del cordón industrial rosarino. Al señalar la estrecha relación entre acceso al crédito y formas de consumo, el artículo evidencia el modo en que estas prácticas están relacionadas con la manera en que las personas se proyectan en el futuro. Si por un lado el acceso al crédito se relaciona con la posibilidad de una vida mejor y representar formas de progreso (refacción de las casas o adquirir herramientas de trabajo), también deja atado el futuro al cumplimiento de las deudas contraídas. Las aspiraciones e imaginarios de futuro también performan las concepciones del delito como forma de vida, en una proyección del porvenir modelado por imperativos sociales y culturales que analiza Pablo Figueiro. En esas formas de ganarse la vida, siguiendo al autor, en los cálculos e inversiones a futuro entra en juego la familia, principalmente. Las aspiraciones a futuro como formas de autonomía y de progreso son igualmente significativas en el análisis de las trayectorias de las y los costureros que propone Dolores Señorans. Los recorridos migratorios que los conducen a instalarse en el Gran Buenos Aires o a organizarse en cooperativas dentro del Movimiento de Ttrabajadores Excluidos (MTE) cobran sentido en el marco de expectativas y proyecciones a futuro que la autora define como plurales, y tensionan dicotomías entre beneficio individual y colectivo. La apuesta por la construcción de una organización gremial incluye esas expectativas plurales en un proceso de lucha colectiva por una vida mejor. En el caso de los vendedores ambulantes que aborda Pires, son tanto las motivaciones económicas, 
personales y de reconocimiento social, como lecturas posibles sobre el derecho que los actores hacen las que marcan esas prácticas hacia el futuro.

Así, la incorporación de un análisis atento a las proyecciones u orientaciones a futuro resulta fundamental en la medida en que estas performan la vida en el presente y están modeladas por experiencias pasadas en una temporalidad que anuda pasado-presentefuturo. Lejos de constituir una suerte de futurología, las reflexiones recientes sobre el futuro como campo de indagación antropológico se afirman en un análisis del futuro en el presente (Pels, 2015; Pink y Salazar, 2017; Bryant y Knight, 2019; Jansen; 2019).

En consecuencia, tal como ha sido señalado por esta literatura, se trata de una pregunta que supone una reflexión sobre la temporalidad, que interroga una concepción lineal de tiempo para incorporar el sentido indeterminado, múltiple y plural en relación con el futuro como posibilidad. Se trata así de una reflexión que habilita una perspectiva crítica al incorporar las apuestas y búsquedas por otras formas de vivir. En esta clave podemos leer el análisis que propone Julieta Gaztañaga sobre las maneras en que un movimiento social vasco practica el derecho a decidir como parte de un proyecto social alternativo de producción de soberanía del activismo vasco frente a la monstruosidad de la violencia estatal. La posibilidad de parir en euskera es, sin duda, una imagen sumamente potente del sentido de esta afirmación soberana, en tanto apuesta por generar las condiciones para decidir sobre la forma en que se producen vidas que valen la pena ser vividas. El análisis sobre la relación entre la temporalidad de las finanzas y la del trabajo que propone Hadrien Saiag evidencia no solo el modo en que el futuro modela el presente, sino que también habilita a una crítica sobre las formas de alienación y explotación a la que los sectores populares están expuestos.

\section{Coda}

Escribimos la introducción a este dossier en tiempos de la pandemia de la COVID-19 y-en nuestro caso- de aislamiento social preventivo y obligatorio. Durante estos meses, decenas de textos fueron escritos por investigadoras e investigadores de diferentes disciplinas. Los procesos actuales de crisis nos han llevado a una situación inédita -al menos en las últimas décadas- que ha afectado todas las esferas de la vida. Parece hoy difícil hablar de otra cosa que no sea de la COVID-19 y sus efectos en la cotidianidad y en el futuro. Es innegable que las tres líneas que planteamos aquí -valor, futuro y trabajo- están puestas en debate a partir de esta crisis que afecta a casi todos los países del planeta. El aislamiento y la pandemia han evidenciado de manera muchas veces dramática las desigualdades sociales y la precarización de grandes sectores de la población (tanto por sus condiciones habitacionales, como por la necesidad de trabajar para vivir o la necesidad de obtener recursos por no poder trabajar), así como el rol de los Estados y de la política pública para construir formas de prevención a la vez que tienden a favorecer a ciertos grupos sobre otros.

La pandemia también ha puesto de relieve las discusiones sobre cómo se produce una vida que vale la pena ser vivida. Ha demostrado que las maneras en que se constituyen las formas dignas de vivir pueden pensarse como plurales. Esto es, existen diferentes valoraciones sobre cómo desarrollar su vida cotidiana en condiciones en que aquello que consideramos parte de la normalidad parece estallar. A su vez, las imaginaciones de un futuro diferente muestran el poder de esta crisis -como ha planteado Federico Neiburg (2020) - por poner en pausa las formas de vivir. Así, pensar en el futuro como una producción de sentidos no implica hacer futurología sobre lo que vendrá, sino comprender cómo las personas piensan sus vidas en este contexto. Y sobre todo, merecen ser objeto de investigación, no como algo que ocurrirá, sino como apuestas a formas de vivir. Son formas presentes de vivir el futuro. Cuando analizamos formas 
de (ganarse la) vida, estamos pensando en formas de poder y formas de (buscar) imponer órdenes morales. La pandemia ha hecho "más evidente" que, ante procesos de incertidumbre, es posible apreciar los marcos que guían la acción y las múltiples formas de otorgar valor a la vida.

\section{Sobre los autores}

Maria Inés Fernández Álvarez, doctora en Antropología (UBA), profesora adjunta del Departamento de Antropología (UBA), investigadora independiente del CONICET con sede en el Instituto de Ciencias Antropológicas.

Mariano Perelman, doctor en Antropología (UBA), jefe de Trabajos Prácticos del Departamento de Antropología (UBA), investigador adjunto del CONICET con sede en el Instituto Gino Germani. 


\section{Q Referencias bibliográficas}

» Adler de Lomnitz, L. (1975). Cómo sobreviven los marginados. Buenos Aires: Siglo XXI.

»Álvarez Leguizamón, S. (2008). Pobreza y desarrollo en América Latina. Salta: Universidad Nacional de Salta.

» Benería, L. (1987). ¿Patriarcado o sistema económico? Una discusión sobre dualismos metodológicos. En C. B. Amorós (ed). Mujeres: ciencia y práctica política (pp. 39-52). Madrid: Debate.

» Bryant, R. y Knight, D. (2019). The Anthropology of the Future. Cambridge: Cambridge University Press.

»Cardoso, F. H. y Faletto, E. (1969). Dependencia y desarrollo en América Latina. Ensayo de interpretación sociológica. México: Siglo XXI.

» Carrasco, C. (2013). El cuidado como eje vertebrador de una nueva economía. Cuadernos de Relaciones Laborales, 31(1), 39-56.

»Carrier, J. y Kalb, D. (2015). Anthropologies of Class. Power, practice and inequality. Cambridge: Cambridge University Press.

»Chua, L. y Fair, H. (2019). Anthropocene. Cambridge Encyclopedia of Anthropology. Recuperado de https://www.anthroencyclopedia.com/entry/anthropocene

»Comas D’Argemir, D. (1015). Los cuidados de larga duración y el cuarto pilar del sistema de bienestar. Revista de Antropología Social, 24, 375-404.

»Cosacov, N. y Perelman, M. D. (2015). Struggles over the Use of Public Space: Exploring Moralities and Narratives of Inequality. Cartoneros and Vecinos in Buenos Aires. Journal of Latin American Studies, 47(03), 521-542.

»Fernández Álvarez, M. I. (2020a). Building from heterogeneity: The decomposition and recomposition of the working class viewed from the "popular economy" in Argentina. Dialectical Anthropology, 44, 57-68.

»Fernández Álvarez, M.I. (2020b). Para una afirmación etnográfica de la noción de clase social: reflexiones a partir de un estudio con trabajadores de la "economía popular" en Argentina. En H. Palermo y L. Capogrossi (eds). Tratado Latinoamericano de Antropología del Trabajo. Buenos Aires: Consejo Latinoamericano de Ciencias Sociales/Centro de Estudios e Investigaciones y Laborales-Consejo Nacional de Investigaciones Científicas y Técnicas/ Centro de investigaciones y Estudios sobre Cultura y Sociedad- Consejo Nacional de Investigaciones Científicas y Técnicas-Universidad Nacional de Córdoba.

»Fernández Álvarez, M. I. (2018). Más allá de la precariedad: Prácticas colectivas y subjetividades políticas desde la economía popular argentina. Íconos - Revista de Ciencias Sociales, 62, 21-38.

»Fernández Álvarez, M. I. (2017). La política afectada. Experiencia, trabajo y vida cotidiana en Brukman recuperada. Rosario: Prohistoria.

»Fernández Álvarez, M. I. (2016). Experiencias de precariedad, creación de derechos y producción colectiva de bienestar(es) desde la economía popular. Ensambles, 4/5, 72-89.

» Fernández Alvarez, M.I, Wolanski, S., Señorans, D., Pacfico, F., Pederiva, C., Laurens, M.P., Sciortino, S., Sorroche, S., Taruselli, S. y Cavigliaso, C. (2019) Bajo Sospecha. Debates urgentes sobre las clases trabajadoras en Argentina. Buenos Aires: Callao Cooperativa Cultural. 
»Frederici, S. (2010). Calibán y la bruja. Mujeres, cuerpo y acumulación primitiva. Madrid: Traficantes de Sueños.

» Germani, G. (1973). El concepto de marginalidad. Significado, raíces históricas y cuestiones teóricas, con particular referencia a la marginalidad urbana. Buenos Aires: Nueva Visión.

» González de la Rocha, M. (2004). De los recursos de la pobreza a la pobreza de recursos y a las desventajas acumuladas. Latin American Research Review, 39(1), 192-195.

"Graeber, D. (2018). Hacia una teoría antropológica del valor. La moneda falsa de nuestros sueños. Trad. J. Gaztañaga. Buenos Aires: Fondo de Cultura Económica.

» Gregory, C. A. (2015). Preface to the second edition. En Gifts and commodities (pp. xix-li). Chicago: HAU Books.

" Gunder Frank, A. (1970). Capitalismo y subdesarrollo en América Latina. México: Signos.

» Guyer, J. I. (2016). Legacies, Logics, Logistics. Chicago: The University of Chicago Press.

» Hart, K. y Ortiz, H. (2014). The Anthropology of Money and Finance: Between Ethnography and World History. Annual Review of Anthropology, 43(1), 465-482.

» Heywood, P. (2017). The Ontological Turn. Cambridge Encyclopedia of Anthropology. Recuperado de https://www.anthroencyclopedia.com/entry/ontological-turn

» Jansen, S. (2019) Yearnings: On Keeping the Present and the Past at the Heart of an Anthropology of the Future. En R. Bryant y D. M. Knight (Eds.), Orientations to the Future. Recuperado de http://americanethnologist.org/features/collections/orientations-tothe-future/yearnings-on-keeping-the-present-and-the-past-at-the-heart-of-an-anthropology-of-the-future

»Kashmir, S. y Carbonella, A. (2008). Dispossession and the Anthropology of Labor. Critique of Anthropology, 28(5): 5-25.

» Kohn, E. (2015). Anthropology of Ontologies. Annual Review of Anthropology, 44(1), 311-327.

» Lambek, M., Das, V., Fassin, D. y Keane, W. (2015). Four lectures on ethics: Anthropological perspectives. Chicago: Hau.

» Lazar, S. y Sanchez, A. (2019) Understunding labour politcs in an age of precarity. Dialectical Anthropology, 43, 3-14.

» Marini, R. M. (1973). Dialéctica de la dependencia. México: Era.

" Mathews, A. S. (2020). Anthropology and the Anthropocene: Criticisms, Experiments, and Collaborations. Annual Review of Anthropology, 49(1), 67-82.

» Maurer, B. (2006). The Anthropology of Money. Annual Review of Anthropology, 35(1), 1536.

» Millar, K (2018). Reclaiming the Discarded. Life and Labor on Rio's Garbage Dump. Durham: Duke University Press.

» Narotzky, S. (2016). Between Inequality and Injustice: Dignity as a Motive for Mobilization During the Crisis. History and Anthropology, 27(1), 74-92.

» Narotzksy, S. (2018) Rethinking the concept of labour.Journal of the Royal Anthropological Institute, 24, N S1, 29-43.

» Neiburg, F. (2010). Os Sentidos Sociais da Economia. En L. F. Dias Duarte (Ed.). Horizontes das Ciências Sociais no Brasil (pp. 225-258). São Paulo: ANPOCS/Barcarolla/Discurso Editorial.

» Neiburg, F. y J. Guyer (2019). Introduction. The Real y the Real Economy. En F. Neiburg y J. Guyer (eds.). The Real Economy (pp. 1-25). Chicago: Hau Books. 
》 Neiburg, F.(2020). Vidas, economia e emergência. Boletin, Cientistas Sociaiseo Coronavírus, ANPOCS, 22. Recuperado de http://anpocs.org/index.php/publicacoes-sp-2056165036/ boletim-cientistas-sociais/2335-boletim-n-22-vidas-economia-e-emergencia?idU=1

»Nun, J. (2001). Marginalidad y exclusión social. Buenos Aires: Fondo de Cultura Económica.

»Parry, J. P., y Bloch, M. (Eds.). (1989). Money and the morality of exchange. Cambridge: University Press.

»Pels, P. (2015). Modern Times Seven Steps toward an Anthropology of the Future Author(s). Current Anthropology, 56(6), 779-796.

"Perelman, M. D. (2015). Viejos y nuevos cirujas. Construcciones temporales y espaciales en la Ciudad de Buenos Aires. Cuadernos de antropología social, 42, $125-141$.

»Perelman, M. D. (2016). Contesting Unemployment: The case of the cirujas in Buenos Aires. En J. B. Kwon y C. M. Lane (Eds.), Anthropologies of Unemployment: New Perspectives on Work and Its Absence (pp. 97-117). Ithaca: Cornell University Press.

»Perelman, M. D. (2017). Trabalho, temporalidade e processos coletivos. Transformações nas subjetividades de vendedores ambulantes e catadores informais. Trabalho (En)Cena, 2(2), 83-97.

»Perelman, M. D. (2020). Para una antropología amplia del trabajo desde y en Argentina. En H. Palermo y L. Capogrossi (eds). Tratado Latinoamericano de Antropología del Trabajo. Buenos Aires: Consejo Latinoamericano de Ciencias Sociales, /Centro de Estudios e Investigaciones y Laborales-Consejo Nacional de Investigaciones Científicas y Técnicas/ Centro de investigaciones y Estudios sobre Cultura y Sociedad- Consejo Nacional de Investigaciones Científicas y Técnicas- Unviersidad Nacional de Córdoba.

"Perelman, M. D. y Boy, M. (Eds.). (2017). Fronteras en la ciudad:(Re)producción de desigualdades y conflictos. Buenos Aires: Teseo.

"Pérez Orozco, A. (2014). Subversión feminista de la economía. Aportes para un debate. Madrid: Traficantes de Sueños.

»Pink, S. y Salazar, F. (2017). Anthropologies and Futures: Setting the Agenda En F. Sala$z a r$, S. Pink y A. Irving (Eds.). Anthropologies and Futures: researching emerging and uncertain worlds (pp. 3-22). Oxford: Bloomsbury

》Quijano, A. (2014). Cuestiones y horizontes: De la dependencia histórico-estructural a la colonialidad/descolonialidad del poder: antología esencial (Primera edición). Buenos Aires: CLACSO.

" Robbins, J. y Stein, F. (2016). Values. Cambridge Encyclopedia of Anthropology. Recuperado de https://www.anthroencyclopedia.com/entry/values

"Sanchez, A. (2018). The Fall and Rise of Class. En H. Wydra y B. Thomassen (Eds.). The Handbook of Political Anthropology (pp. 410-422). Cheltenham: Edward Elgar Publishing.

»Saraví, G. (2007) De la pobreza a la exclusión. Continuidades y rupturas de la cuestión social en América Latina. Buenos Aires: Prometeo.

»Visacovsky, S. (2019). Futuros en el presente. Los estudios antropológicos de las situaciones de incertidumbre y esperanza. PUBLICAR-En Antropología y Ciencias Sociales, 26, 6-25.

"Wolanski, S. (2020) (Re)produire le syndicat, produire la classe : la formation de la classe ouvrière dans un syndicat argentin. Critique internationale, 86(1), 167-188. 